\title{
Design, Optimization and Simulation of Knee Pain Relief Device Using Hyperthermia Technology
}

\author{
Saman Rajabi, Ramin Akbari Asbagh ${ }^{*}$, Faraz Askarizadegan \\ Faculty of Electrical Engineering, Siraj Institute of Higher Education, Tabriz, Iran \\ Email address: \\ s.rajebi@seraj.ac.ir (S. Rajebi), ramin.akbari.asbagh@gmail.com (R. A. Asbagh), farazaskarizadegan@gmail.com (F. Askarizadegan) \\ ${ }^{*}$ Corresponding author
}

\section{To cite this article:}

Saman Rajabi, Ramin Akbari Asbagh, Faraz Askarizadegan. Design, Optimization and Simulation of Knee Pain Relief Device Using Hyperthermia Technology. American Journal of Electromagnetics and Applications. Vol. 8, No. 2, 2020, pp. 40-45.

doi: 10.11648/j.ajea.20200802.12

Received: October 3, 2020; Accepted: October 19, 2020; Published: October 26, 2020

\begin{abstract}
Hyperthermia has been introduced as a therapeutic method and adjuvant therapy in the treatment of some diseases such as breast and pancreatic cancers. On the other hand, other applications of hyperthermia have been considered. In this article, ways to treat knee pain caused by osteoarthritis, patellar chondromasia, rheumatism, knee infection, etc., have been studied using antenna hyperthermia. For antenna hyperthermia, is using a rectangular microstrip antenna, which is the simplest type of antenna. The antenna considered in this article has conformal conditions and is designed in the shape of a knee structure, to create a suitable knee covering. The antenna considered in this article has conformal conditions and is designed in the shape of a knee structure, to create a suitable knee covering. In addition to the geometric structure of the antenna, which fits the structure of the knee, the resonance frequency of the antenna is in the range of ISM standard frequencies and attempts have been made to place it at $2.4 \mathrm{GHz}$. On the other hand, to show the thermal pattern created by the antenna, the specific absorption rate of the tissue is investigated and its diagram is simulated by the antenna radiation field.
\end{abstract}

Keywords: Knee Pain, Hyperthermia, Microstrip Antenna, Conformal

\section{Introduction}

Knee pain is one of the most common musculoskeletal problems among people. In today's increasingly active society, the number of knee problems has increased and knee pain has a wide range of specific causes and treatments. The main function of the knee joints is to bend and straighten, and to support the weight of the body along with the ankle and pelvis. The knee joint has the most rotation and flexion to perform all of these movements and support the entire body, while doing so relies on a number of bones, ligaments, tendons, and cartilage [1].

Symptoms of knee pain include redness and burning sensation in the knee and difficulty walking due to pain and discomfort and inability to extend the knee and lock the knee (inability to bend the knee) and swelling and dryness and stinging. Knocking or ringing of the

knee and tenderness of the knee. Chronic knee pain has a variety of causes, including osteoarthritis, Schlatter's disease, patellar chondromalacia, rheumatism, knee bursitis, and infection [2].

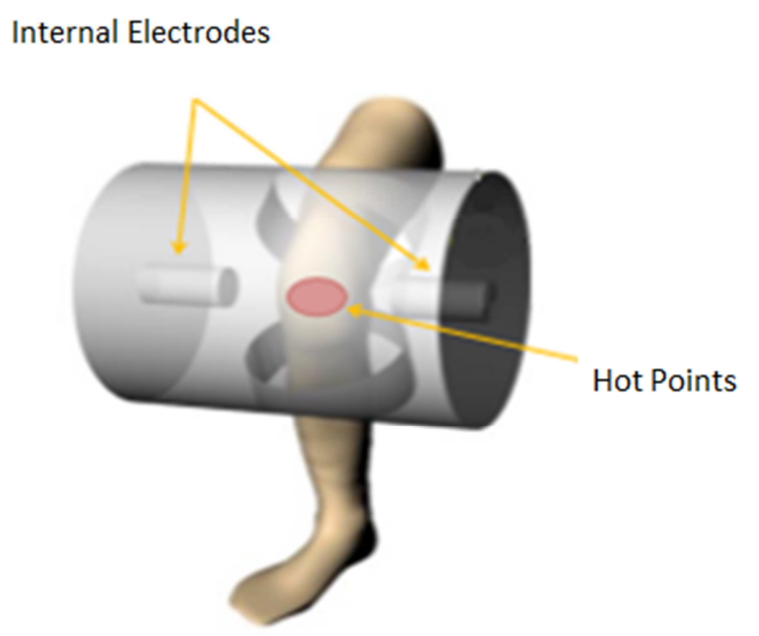

Figure 1. Knee heating system using electric cathodes.

One of the most common methods of treating physical and 
even mental illnesses is heat therapy. High body temperature usually causes illness (such as fever or heat shock). But controlled temperature rise is used to treat diseases such as cancer. A controlled rise in temperature increases blood collagen and reduces joint dryness, pain and muscle spasm. This type of heat therapy is known as hyperthermia [3].

Previous methods of heating the tissue (hyperthermia) were possible by direct heat application. Direct application of heat to the tissue will cause hot spots on the skin of the tissue under heat. Blisters and severe burns are the disadvantages of this method. An example of these methods can be seen in Figure 1.

In order to control the place of temperature centralization and prevent hot spots on the skin has been considered the use of electromagnetic waves to heat the tissue. The term hyperthermia is now used to describe heat using electromagnetic waves. According to this definition, hyperthermia means an increase in the temperature of a part of the body tissue above its normal level. Heat treatment is a process that involves raising the temperature of tumorcontaining tissues, usually up to more than $42^{\circ} \mathrm{C}$. The ability to control the distribution of energy within living tissues with the proliferation of electronic devices and modeling systems has been greatly enhanced in recent decades $[4,5]$.

Kazunaki et al. using the finite element method, the heat properties of the resonance cavity applicator are described with an extended 3D model of the anatomical human knee. The results showed that deep space heating methods were useful in the treatment of knee joint. The researchers also reviewed knee experiments using a new heating rehabilitation system. Warming the tissue deep inside the knee joint is essential for effective thermal rehabilitation of osteoarthritis. The newly studied rehabilitation system is the resonant cavity created in previous studies for the treatment of profound hyperthermia. Experimental results using phantom agar showed that our heating system is able to heat deep tissue inside the knee without physical contact with the surface [6-8].

In this article, a microstrip antenna will be used to create optimal hyperthermia on the knee joints. Therefore, first, the design basis of a simple rectangular microstrip antenna is examined. Then, taking into account the necessary considerations, the simple antenna designed to fully cover the knee is redesigned in the form of a conformal. The effect of knee tissue on antenna resonance will be fully considered. Finally, the warming of the knee tissue is examined.

\section{Research Method and Findings}

\subsection{Rectangular Microstrip Antenna Design}

The concept of microstrip antenna was first introduced by Deshan in late 1953. Over the past few decades, extensive research has been done on the structure of these antennas. In 1970, the first microstrip antenna was developed by Howell and Monson [9]. The microstrip antenna is commonly referred to as an insulating structure, on one side, on the ground and on the other side of the radiation plate. This conductor is designed in various forms, usually made of copper or gold.

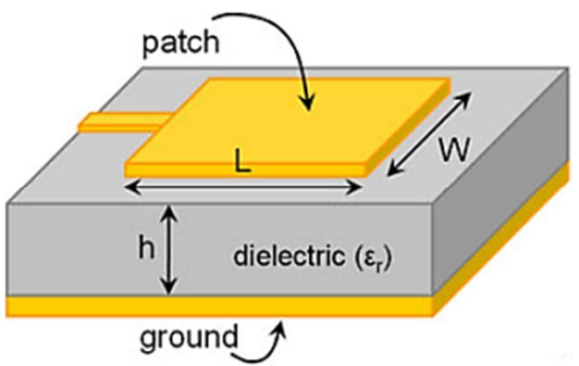

Figure 2. Microstrip antenna geometry with microstrip feed line.

Some of the methods of analysis and design of microstrip antennas are transmission line method, amplifier method and whole wave method. An example of this type of antenna is shown in Figure 2, where the rectangular patch antenna is powered by a microstrip line.

The three basic parameters in the design of microstrip patch antennas are resonant frequency $(f o)$, dielectric constant ( $\varepsilon r)$ and dielectric bed height. Usually the height $(h)$ and the loss tangent $(\tan \delta)$ of the bed are considered in small values. First, the patch width is determined by Equation (1):

$$
W=\frac{c}{2 f_{o} \sqrt{\frac{\left(\varepsilon_{r}+1\right)}{2}}}
$$

Where, $c$ is the speed of light, $\varepsilon_{r}$ is the relative dielectric constant of the substrate and $f_{0}$ is the optimal frequency [10, $11]$.

The three resonant frequencies allowed for hyperthermia applications are: $434 \mathrm{MHz}, 915 \mathrm{MHz}$ and $2.4 \mathrm{GHz}$. In this paper, the resonance frequency of $2.4 \mathrm{GHz}$ is selected and the patch width is determined using FR4 as the substrate $\left(\varepsilon_{r}=4.5\right)$.

Part of the electric field radiated from the patch exits the dielectric bed and passes through a path in the air before reaching the dielectric bed (Figure 3). This phenomenon, which occurs as a result of leakage fields, causes the dielectric constant to decrease and is called the effective dielectric constant, since the propagation medium of this part of the electric field does not occur only inside the substrate [12].

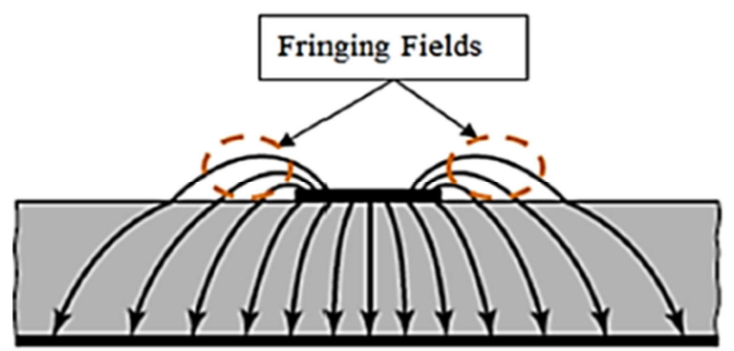

Figure 3. Display of leak fields in microstrip antennas.

Due to these marginal fields, the length of microstrip patch antennas is considered to be longer than their physical length. This length is denoted by $\Delta L$, which is represented by the following equation: 


$$
\Delta L=0.412 h \frac{\left(\varepsilon_{\text {reff }}+0.3\right)\left(\frac{w}{h}+0.264\right)}{\left(\varepsilon_{\text {reff }}-0.258\right)\left(\frac{w}{h}+0.8\right)}
$$

Where, $w$ is the width of patch, $h$ is the height and $\varepsilon_{\text {reff }}$ is the relative dielectric Constant of the substrate respectively. Due to this increase in the electrical length of the patch, the physical length of the patch is found by Equation (3).

$$
L_{\text {eff }}=\frac{c}{2 f_{o} \sqrt{\varepsilon_{\text {reff }}}}-2 \Delta L ?
$$

In this paper, the patch is performed by connecting the electric and magnetic fields of the transmission line. In this design, the characteristics of the microstrip path antenna designed with FR4 substrate are: dielectric constant $\varepsilon_{r}=4.7$, dielectric substrate dissipation coefficient $\tan \delta=0.025$. The dielectric bed thickness is considered to be $1.6 \mathrm{~mm}$ and the patch thickness is considered to be $2 \mathrm{~mm}$. Also, the length and width of the patch are equal to 5 and $4 \mathrm{~cm}$, respectively. According to the considered dimensions, the return drop curve of the microstrip antenna is shown in Figure 4.

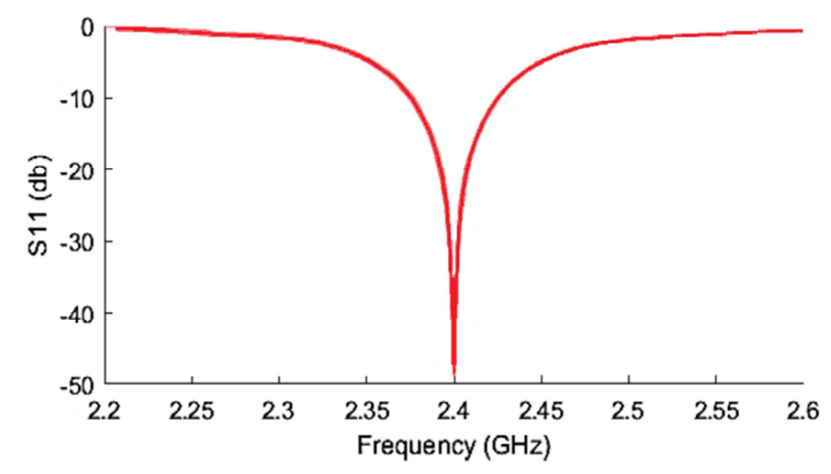

Figure 4. Curve $S_{11}$ Simple rectangular microstrip antenna.

Despite the simple design of the rectangular microstrip antennas, the inconsistency of its physical shape with the intended application will be problematic. For this purpose, in order to make it possible to match the maximum antenna with the knee structure, the designed antenna should be designed in conformal conditions.

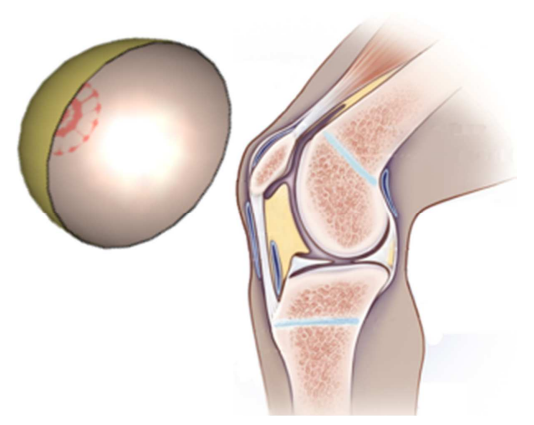

Figure 5. Selecting a hemispherical conformal structure for the antenna to properly cover the knee.

\subsection{Microstrip Conformal Antenna Design}

According to Figure 5 , in order for the antenna to properly cover the knee, the microstrip antenna must be designed conformably in the form of a hemispherical structure.

Figures 6 and 7 show the structure of the conformal antenna. The wave number of a microstrip antenna can be defined as Equation (4) [13]

$$
K_{\text {eff }}=K_{0} \sqrt{\varepsilon_{r}\left(1-j \tan \delta_{e f f}\right)}
$$

Where, $K_{0}$ is the wave number in the free space and $\tan \delta_{\text {eff }}$ is the effective loss tangent. The effective loss tangent can be shown as Equation (5).

$$
\tan \delta_{\text {eff }}=\frac{1}{Q}
$$

$Q$ Indicates the quality coefficient of the designed conformal microstrip antenna. The quality coefficient of the conformal microstrip antenna designed can be defined by the following relation:

$$
Q=\left[\frac{15 \lambda^{2}(W+\Delta W) \varepsilon_{r}}{136 \sqrt{\varepsilon_{r}-1} \cdot h^{2}(L+\Delta L)}+\frac{1}{\pi h} \sqrt{\frac{\lambda}{120 \sigma_{C}}}+\tan \delta\right]^{-1}
$$

Where $h$ and $\sigma_{c}$ are the height and the specific conductance of the used substrate respectively. Also, $\lambda$ indicates the used wave length for the antenna. According to the geometric structure of the conformal microstrip antenna given in Figure 6, the perpendicular angles can be determined using the equations (7) and (8):

$$
\begin{gathered}
\varphi_{0}=\frac{W-\Delta W}{R} \\
\varphi_{1}=\frac{L-\Delta L}{R}
\end{gathered}
$$

Where $\mathrm{R}$ is the inner radial of the conformal substrate. The length and width of the mentioned conformal patch are determined according to the relationships of conventional microstrip antennas. Of course, it should be borne in mind that the resonant bandwidth of the antenna will also change compared to a simple rectangular microstrip antenna, which is calculated from the following equation:

$$
B W=\frac{1}{\sqrt{2} Q} \times 100 \%
$$

After reviewing the design theory of rectangular microstrip antennas and then the conformal conditions for them, the conformal antenna is designed according to Figure 7. For antenna resonance at $2.4 \mathrm{GHz}$, the following specifications are considered for the design:

Patch material: copper

Substrate material: FR4

Ground layer material: copper

Patch thickness: $0.07 \mathrm{~mm}$

Substrate thickness: $0.47 \mathrm{~mm}$ 
Ground layer thickness: $0.07 \mathrm{~mm}$

Internal radius of the hemisphere: $36 \mathrm{~mm}$

With the necessary calculations for the antenna dimensions to operate at the desired frequency, its $\mathrm{S}_{11}$ diagram is obtained as Figure 8. The antenna conditions for operation at a frequency of $2.4 \mathrm{GHz}$ are well evaluated, according to the allowable resonant frequency range. The antenna bandwidth also shows a relative improvement over simple rectangular microstrip antennas that are very narrow.

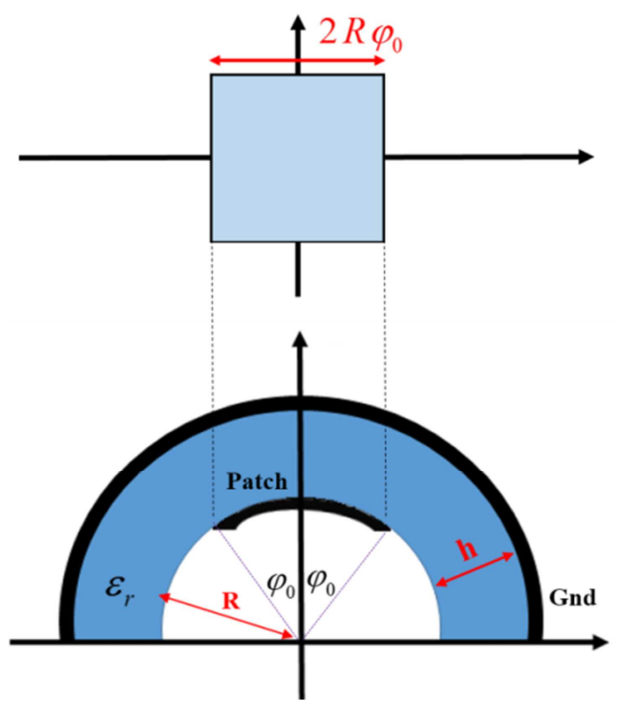

Figure 6. Geometric structure of designed conformal antenna.

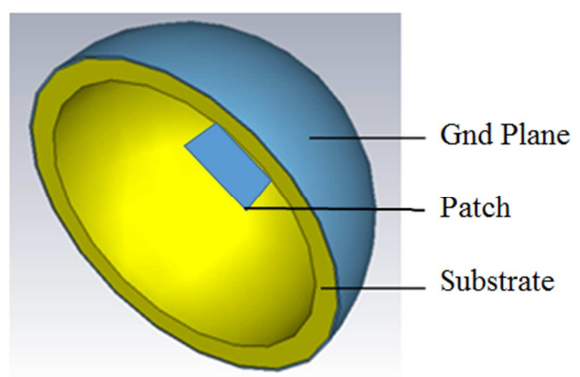

Figure 7. Conformal patch antenna view designed in CST environment.

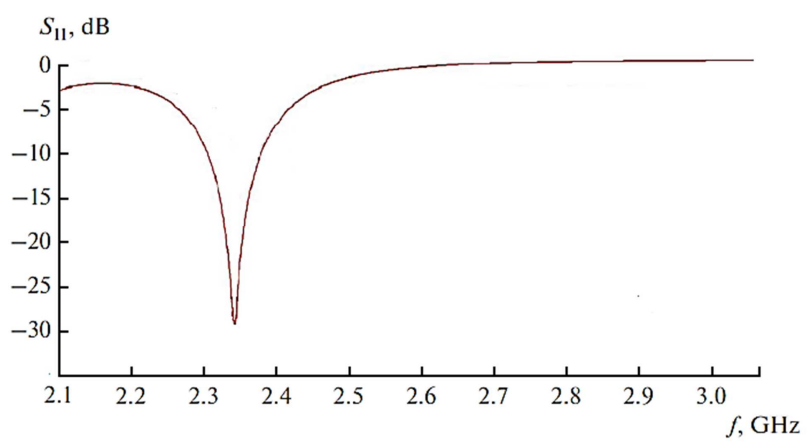

Figure 8. $S_{11}$ Diagram of Conformal antenna shown in Figure 7.

To evaluate the effect of the substrate used in the design of the conformal antenna, have been changed the material and thickness of the substrate and the effects of these changes in the antenna resonance frequency are reported according to the diagrams shown in Figures 9 and 10.

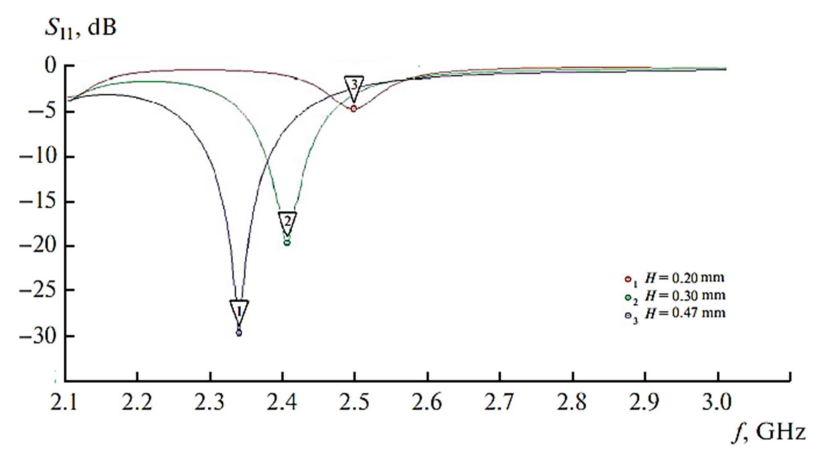

Figure 9. Effect of substrate thickness changes on antenna resonant frequency.

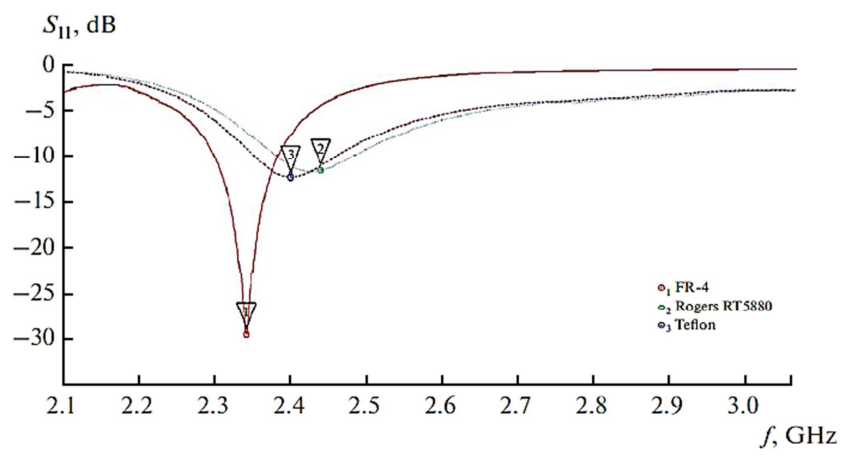

Figure 10. Effect of substrate change on antenna resonance frequency.

According to the $\mathrm{S}_{11}$ diagrams presented in Figures 9 and 10 , the best substrate material and the best substrate thickness can be considered as FR4 as $0.47 \mathrm{~mm}$, respectively.

\section{The Specific Absorption Rate}

To measure the amount of heat produced in the tissue by an antenna, is used a parameter called the specific absorption rate. The specific absorption rate indicates the amount of radiant energy emitted by the antenna and absorbed in different layers of body tissue and at different depths of the skin. The SAR value depends on the effect of the internal electric field [14, 15]. Several relationships can be used to measure the SAR value. According to the first relation, SAR is equal to the rate of energy dissipation in a tissue with mass ' $m$ '.

$$
S A R=\frac{d}{d t} \frac{d w}{d m}=\frac{d}{d t} \frac{d w}{\rho d v}
$$

Where, $\rho$ and $v$ are equal to the volumetric mass and the volume of the desired mass, respectively. It can also be called SAR according to the rate of increase of tissue temperature that is defined as the following relation:

$$
S A R=C_{h} \frac{d T}{d t}
$$

Where, $C_{h}$ is the specific heat capacity of the desired tissue. Also, the specific absorption rate can be expressed in terms of the electric field penetrated into the tissue, specific 
conductivity and tissue volume density as follows:

$$
S A R=\frac{\sigma E^{2}}{\rho}
$$

According to the equations expressed for SAR, it can be seen that with the penetration of an electric field into the tissue, the tissue temperature begins to increase in terms of conductivity and density of the desired area of the tissue as well as its specific heat capacity. Therefore, to study the amount of heat produced in the knee tissue, the knee tissue must first be simulated. The anatomy of the knee is shown in Figure 11a and the proposed model for it in CST software is shown in Figure 11b.

According to the approximate model for the knee according to Figure $11 \mathrm{~b}$, its thermal simulation was performed with a designed microstrip conformal antenna and the simulation result was obtained as shown in Figure 12.

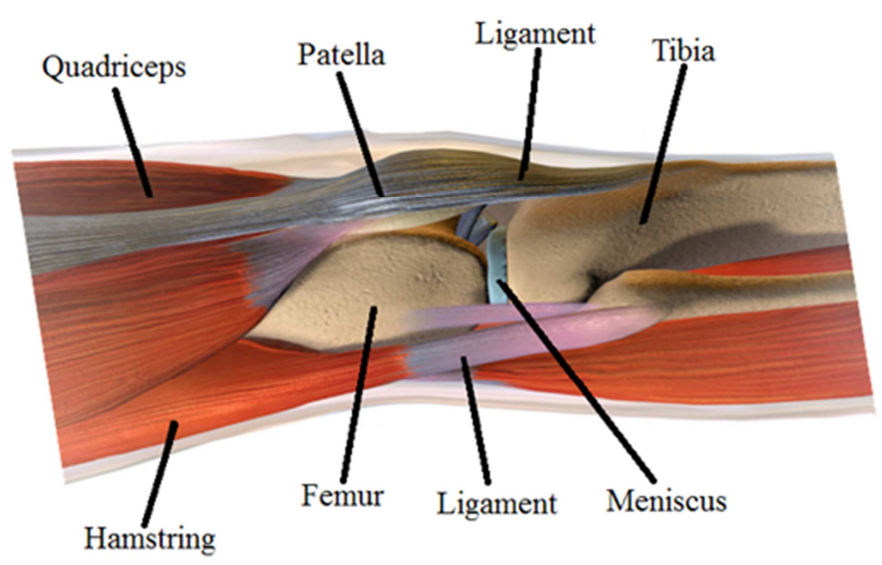

(a)

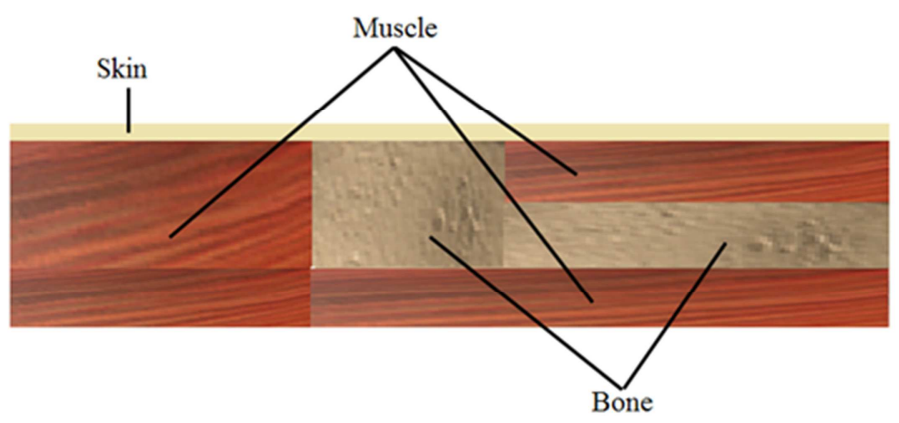

(b)

Figure 11. A: The true anatomy of the knee. B: Knee modeled for CST software.

According to the approximate model for the knee according to Figure $11 \mathrm{~b}$, its thermal simulation was performed with a designed microstrip conformal antenna and the simulation result was obtained as shown in Figure 12.

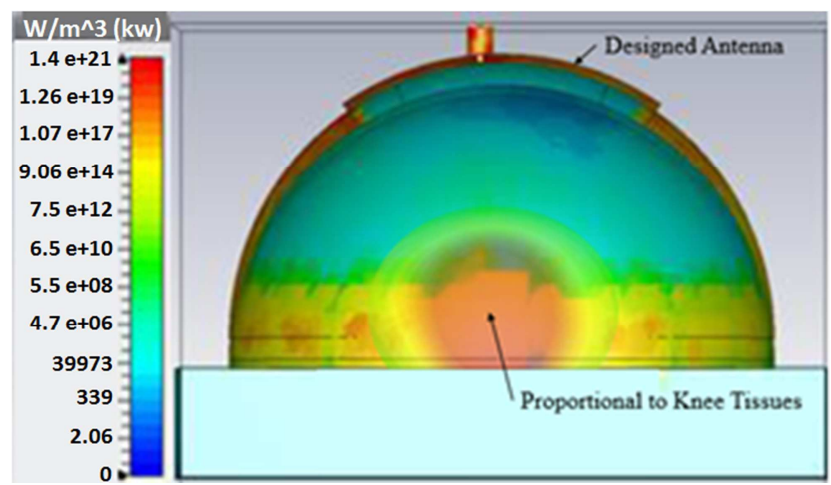

Figure 12. Simulated SAR for the knee model considered in Figure $12 b$.
According to the SAR diagram shown in Figure 12, its functional accuracy can be ensured at a resonant frequency of $2.4 \mathrm{GHz}$ as well as heat generation in the knee area.

\section{Conclusion}

In this article, one of the ways to treat knee pain (due to arthritis, patellar chondromasia, rheumatism, knee infection, etc.) was evaluated using hyperthermia. Microstrip antenna hyperthermia is a new treatment for hyperthermy that is used to treat knee pain by generating thermal energy. In this article, among the types of antennas, microstrip antennas are simulated and analyzed, which are among the simplest, least expensive ways to treat and transfer energy to the desired point in the body. These antennas with low volume and low losses are the best option for heat transfer. For this purpose, a simple rectangular microtrip antenna with a resonant 
frequency at $2.4 \mathrm{GHz}$ was designed. For proper knee coverage, the conformal microstrip antenna was designed with all aspects and its resonance frequency was in the appropriate range. The designed conformal antenna had a very low frequency shift compared to the standard $2.4 \mathrm{GHz}$ frequency. Since a frequency shift of about $5 \%$ usually seems reasonable, the design will be acceptable.

Finally, by modeling the anatomical structure of the knee in CST software, created SAR and thermal pattern in the knee tissue has been simulated. The obtained results for SAR show that a well-designed antenna can easily heat the desired area of tissue. High values of SAR in the knee area on the one hand, and low values in other areas of the tissue on the other hand, indicate the proper performance of the antenna in concentrating heat on the target area.

Accurate calculation of temperature in the tissue area and using techniques such as applying materials with a dielectric coefficient close to the tissue dielectric coefficient, to transfer energy into the inner area of the knee tissue with less reflection, can be suitable ideas to continue this work.

\section{References}

[1] X. Yang, J. Du, and Y. Liu, "Advances in hyperthermia technology," in Proc. 2005 IEEE Engineering in Medicine and Biology 27th Ann. Conf., Shanghai, 2005 (IEEE, New York, 2005).

[2] O. Isik, E. Korkmaz, and B. Türetken, "Antenna arrangement considerations for microwave hyperthermia $11 \mathrm{~s}$ applications," in Proc. URSI Gen. Assem. and Sci. Symp., Istanbul, Aug. 13-20, 2011 (IEEE, New York, 2011), pp 1-3.

[3] E. Korkmaz, O. Isik, and M. A. Nassor, "A Compact microstrip spiral antenna embedded in water bolus for hyperthermia applications," Hindawi Publ. Corp. Int. J. Antennas \& Propagtion, ID 954986 (2013).

[4] W. Cheol Choi, K. Joon Kim, H. Soon Park, and Y. Joong Yoon, "Frequency reconfigurable applicator for superficial hyperthermia system," Proc. Isap, Nagoya, Japan, (2012), pp. 26-29.

[5] F. Sheta, I. Elshafiey, and A. Mohra, “A compact antenna for microwave imaging and hyperthermia treatment of brain tumor," in Antenna Technology and Applied Electromagnetics (ANTEM), (Proc. 15th Int. Symp., Toulouse, June 25-28, 2012 (IEEE, New York, 2012).
[6] K. Watanabe, H. Kurosaki, Mitsunori Kubo, K. Takahashi, K. Kato, Y. Shindo, Heating properties of resonant cavity applicator for treating rheumatoid arthritis by using 3-D FEM knee model, Computer Science, Engineering, (2013).

[7] Y. Seo Koo, A. Fathy, R. Kazemi, Q. Liu, and J. Phillips, "Development of a high SAR conformal antenna for hyperthermia tumors treatment," IEEE Trans. Antennas Propag. 62, 1401 (2014).

[8] K. J. Kim, W. Cheol Choi, and Young Joong Yoon,"Planar array applicator for the non-invasive local hyperthermia system," in Electromagnetics in Advanced Applications (ICEAA) (Proc. Int. Conf. IEEE APWC, Torino, Italy, Sept. 9-13, 2013) (IEEE, New York, 2013).

[9] S. Curto, P. McEvoy, X. Bao, and Max J. Ammann, "Compact patch antenna for electromagnetic interaction with human tissue at $434 \mathrm{MHz}$, IEEE Trans. Antennas Propag. 57, 2564 2571 (2009).

[10] A Ghasemlouy, S Rajebi, "Investigation and Evaluation of the Effect of Silicon Layer and Its Comparison with Water Bolus in Designing Microstrip Antenna for Hyperthermia Applications", Journal of Communications Technology and Electronics, Volume 64, Issue 11, 2019.

[11] Saman Rajebi, Changiz Ghobadi, Javad Nourinia, Ehsan Mostafapour, " SAR Enhancement of Slot Microstrip Antenna by Using Silicon Layer in Hyperthermia Applications", Wireless Personal Communications journal, Volume 111, issue 3, 2020 .

[12] Saman Rajebi, Changiz Ghobadi and Javad Nooriniya, "Multiport network method and using it for accurate design of square spiral antennas", Proceedings of the 5th WSEAS International Conference on Software Engineering, Parallel and Distributed Systems, Pages 180-186, 2006.

[13] Liu P., Li N., Huang H. "Error Modeling Analysis of Conformal Microstrip Antenna.", Proceedings of the Seventh Asia International Symposium on Mechatronics. Lecture Notes in Electrical Engineering, vol 588. Springer, Singapore. 2020. https://doi.org/10.1007/978-981-32-9437-0_26

[14] Mahsa Jahangiri, Saman Rajebi, "Effects of Split Ring Resonator (SRR) Metamaterial on the Radiation Pattern and Variation of the Heating Focus Point of the Microstrip Patch Antenna", ASTES Journal, Volume 5, Issue 1, 2020.

[15] M. Salimi, Sh. Gheitarani Sehrigh, S. Rajebi, " DESIGN AND ANALYSIS OF MICROSTRIP PATCH ANTENNA FOR HYPERTHERMIA APPLICATIONS IN BREAST CANCER", International Journal on Technical and Physical Problems of Engineering, Volume 11, Issue 41, Pages 71-76, 2019. 